\title{
NUMERICAL STUDY OF A PROBLEM IN THE COMBUSTION OF A POROUS MEDIUM
}

\author{
K. K. TAM and ANDONOWATI ${ }^{1}$
}

(Received 10 December 1994; revised 18 September 1995)

\begin{abstract}
A spectral method is used to consider the porous medium combustion in an infinite slab. The infinite system of ordinary differential equations for the amplitude functions is truncated and comparisons are made for different numbers of modes included in the numerical computation. It is shown that the qualitative behaviour of the solution is captured by the first eigenmode. Dependence of the solution on initial data and a parameter is also considered.
\end{abstract}

\section{Introduction}

A model for porous medium combustion was proposed by Norbury and Stuart in 1987 [4]. The governing equations are

$$
\begin{aligned}
\frac{\partial \sigma}{\partial t} & =-\lambda r, \\
\sigma \frac{\partial u}{\partial t} & =\frac{\partial}{\partial z}\left\{\left(d u^{3}+1\right) \frac{\partial u}{\partial z}\right\}+w-u+r, \\
\mu \frac{\partial w}{\partial z} & =u-w, \\
\frac{\partial g}{\partial z} & =-\frac{a r}{\mu}, \\
\text { with } \quad r & =H\left(\sigma-\sigma_{a}\right) H\left(u-u_{c}\right) \mu^{1 / 2} g f(w) .
\end{aligned}
$$

The nondimensionalised quantities $\sigma, u$, and $w$ are the heat capacity of the solid, the solid temperature and the gas temperature, respectively, $g$ is proportional to the product of oxygen concentration and gas temperature, $t$ is the time variable and $z$

${ }^{1}$ Department of Mathematics and Statistics, McGill University, 805 Sherbrooke Street West, Montréal, Québec, Canada, H3A 2K6

(C) Australian Mathematical Society, 1997, Serial-fee code 0334-2700/97 
is the space variable. $H(\xi)=0$ if $\xi \leq 0$ and $H(\xi)=1$ otherwise. The function $f(w)$ is usually taken to be proportional to $w^{2}$. The parameter $\mu$ is proportional to the inlet gas velocity while the parameter $\lambda$ is linearly related to the specific heat of the combustible solid. The parameter $\sigma_{a}$ satisfies $0<\sigma_{a}<1$ and $u_{c}$ denotes the critical switching temperature related to the burning zone, that is, a region in the $z$-plane where $r \geq 0$.

In [7], Tam considered the combustion of a porous slab occupying $0 \leq z \leq 1$, using the initial and boundary conditions

$$
\begin{aligned}
\sigma(z, 0) & =\sigma_{s}, \quad \sigma(z, \infty)=\sigma_{a}, \\
u(0, t) & =u(1, t)=0, \quad u(z, 0)=u_{0}(z), \\
w(0, t) & =0 \\
g(0, t) & =g_{a},
\end{aligned}
$$

where $u$ and $w$ have been scaled such that the ambient temperature is zero. The reaction rate $r$ in (5) was also replaced by

$$
r=\left(\sigma-\sigma_{a}\right) \mu^{1 / 2} g w^{2} .
$$

To simplify the problem, Tam [7] used a modified Oseen-type linearization to derive an ordinary differential equation from (1) to (5). It was argued that the information regarding the ignition and the qualitative dependence of the solution on the parameters can be deduced from the ordinary differential equation.

In this paper we study this problem further by expanding the solution in a series of eigenfunctions. The time-dependent coefficients of the eigenfunctions are then governed by an infinite system of ordinary differential equations. To study this system numerically, it must be reduced to a finite system, and we do this simply by using $n$-term truncation. In the next section, are carried out some preliminary manipulations so that only two dependent variables have to be expanded. In Section 3 we focus on the isolated fundamental mode, giving some analysis regarding the initial data dependence of the solution. In Section 4, numerical solutions for the truncated multi-mode systems are obtained and it is shown that results confirm the validity of the qualitative behaviour derived from using a single mode.

To lessen the complexity of the problem we shall take $d=0$.

\section{Solution by eigenfunction expansion}

Let $\chi=\sigma-\sigma_{a}$. Using (10) and integrating (4) with respect to $z$, we have

$$
g(z, t)=g_{a} \exp \left\{-a \mu^{1 / 2} \int_{0}^{z} \chi(s, t) w^{2}(s, t) d s\right\} .
$$


From (3), we obtain

$$
w(z, t)=\frac{1}{\mu} e^{-z / \mu} \int_{0}^{z} u(s, t) e^{s / \mu} d s
$$

Thus if we solve for $u$ and $\chi$, the above results give $g$ and $w$.

Let $\left\{\varphi_{n}\right\}=\{\sqrt{2} \sin n \pi z\}$ be a set of normalized eigenfunctions corresponding to eigenvalues $\left\{\gamma_{n}\right\}=\left\{n^{2} \pi^{2}: n\right.$ is a natural number $\}$. Expand $u$ and $\chi$ in terms of $\left\{\varphi_{n}\right\}$ as

$$
\begin{aligned}
& u(z, t)=\Sigma_{1}^{\infty} U_{n}(t) \varphi_{n}(z) \\
& \chi(z, t)=\Sigma_{1}^{\infty} X_{n}(t) \varphi_{n}(z) .
\end{aligned}
$$

Using the notation

$$
\begin{aligned}
& \bar{\varphi}_{n}(z)=\int_{0}^{z} \varphi_{n}(s) d s \\
& \tilde{\varphi}_{n}(z)=\int_{0}^{z} \varphi_{n}(s) e^{s / \mu} d s
\end{aligned}
$$

and substituting (13) and (14) into (1) and (2), yields

$$
\begin{gathered}
\Sigma_{i} \varphi_{i} X_{i}^{\prime}=-\mu^{-3 / 2} \lambda g_{a} e^{-2 z / \mu} \Sigma_{j} \Sigma_{k} \Sigma_{l} \varphi_{j} \tilde{\varphi}_{k} \tilde{\varphi}_{l} X_{j} U_{k} U_{l} \\
-\mu^{-5 / 2} a e^{-2 z / \mu} \Sigma_{i} \Sigma_{j} \Sigma_{k} \Sigma_{l} \bar{\varphi}_{i} \varphi_{j} \tilde{\varphi}_{k} \tilde{\varphi}_{l} X_{i}^{\prime} X_{j} U_{k} U_{l} \\
\sigma_{a} \Sigma_{i} \varphi_{i} U_{i}^{\prime}+\Sigma_{i} \Sigma_{j} X_{i} \varphi_{i} \varphi_{j} U_{j}^{\prime}= \\
-\Sigma_{i} \gamma_{i}^{2} \varphi_{i} U_{i}+\mu^{-1} e^{-z / \mu} \Sigma_{i} \tilde{\varphi}_{i} U_{i}-\Sigma_{i} \varphi_{i} U_{i}-\lambda^{-1} \Sigma_{i} \varphi_{i} X_{i}^{\prime}
\end{gathered}
$$

where $\Sigma_{n}$ denotes $\Sigma_{n=1}^{n=\infty}$.

Multiplying (17) and (18) by $\varphi_{n}$ and integrating from 0 to 1 with respect to $z$, yields

$$
\begin{aligned}
& X_{n}^{\prime}=-\mu^{-3 / 2} \lambda g_{a} \Sigma_{j} \Sigma_{k} \Sigma_{l} X_{j} U_{k} U_{l} \int_{0}^{1} \varphi_{j} \tilde{\varphi}_{k} \tilde{\varphi}_{l} \varphi_{n} e^{-2 z / \mu} d z \\
& \quad-\mu^{-5 / 2} a \Sigma_{i} \Sigma_{j} \Sigma_{k} \Sigma_{l} X_{i}^{\prime} X_{j} U_{k} U_{l} \int_{0}^{1} \bar{\varphi}_{i} \varphi_{j} \tilde{\varphi}_{k} \tilde{\varphi}_{l} \varphi_{n} e^{-2 z / \mu} d z \\
& \sigma_{a} U_{n}^{\prime}+\Sigma_{i} \Sigma_{j} X_{i} U_{j}^{\prime} \int_{0}^{1} \varphi_{i} \varphi_{j} \varphi_{n} d z= \\
& \quad-\gamma_{n}^{2} U_{n}+\mu^{-1} \Sigma_{i} U_{i} \int_{0}^{1} \tilde{\varphi}_{i} \varphi_{n} e^{-z / \mu} d z-U_{n}-\lambda^{-1} X_{n}^{\prime} .
\end{aligned}
$$


Equations (19) and (20) constitute an infinite-dimensional dynamical system. While it is not possible to solve such a system in general, a great deal of effort has gone into its study. (See, for example, [1], [2], [8].) To reduce it to a finitedimensional system such that at least some numerical work can be done, simplifying assumptions must be made which usually depend on some knowlegde of the underlying problem. In the present case, experience in dealing with this type of problem suggests that the first eigenmode is dominant. We therefore adopt truncation and consider only the interaction of the first $N$ modes, discarding all terms involving modes of order $(N+1)$ and higher. The case of $N=1$ is studied both analytically and numerically. Numerical work on $N=3,5,9,13$ is carried out and the result lends support to the conjecture of the first-mode dominance.

\section{The isolated fundamental mode}

Let $X_{n}$ and $U_{n}$ equal zero for $n \geq 2$. From (19) and (20), dropping the subscript 1 on $X_{1}$ and $U_{1}$, we obtain

$$
\begin{gathered}
X^{\prime}=-\mu^{-3 / 2} \lambda g_{a} C_{1}(\mu) X U^{2}-\mu^{-5 / 2} a C_{2}(\mu) X^{\prime} X U^{2}, \\
\left\{\sigma_{a}+C_{3}(\mu) X\right\} U^{\prime}=-\left(1+\pi^{2}\right) U+\mu^{-1} C_{4}(\mu) U-\lambda^{-1} X^{\prime},
\end{gathered}
$$

where

$$
\begin{aligned}
& C_{1}(\mu)=\int_{0}^{1} \varphi_{1}^{2} \tilde{\varphi}_{1}^{2} e^{-2 z / \mu} d z, \\
& C_{2}(\mu)=\int_{0}^{1} \bar{\varphi}_{1} \varphi_{1}^{2} \tilde{\varphi}_{1}^{2} e^{-2 z / \mu} d z, \\
& C_{3}(\mu)=\int_{0}^{1} \varphi_{1}^{3} d z \\
& C_{4}(\mu)=\int_{0}^{1} \tilde{\varphi}_{1} \varphi_{1} e^{-z / \mu} d z .
\end{aligned}
$$

From (21) we have

$$
X^{\prime}=-\frac{\lambda g_{a} C_{1}(\mu) X U^{2}}{\mu^{2 / 3}+a \mu^{-1} C_{2}(\mu) X U^{2}} .
$$

Substituting (27) into (22), then yields

$$
\begin{aligned}
& \left\{\mu^{2 / 3}+a \mu^{-1} C_{2}(\mu) X U^{2}\right\}\left\{\sigma_{a}+C_{3}(\mu) X\right\} U^{\prime}= \\
& U\left[\alpha(\mu)\left\{1+\mu^{-5 / 2} a C_{2}(\mu) X U^{2}\right\}+g_{a} C_{1}(\mu) X U\right],
\end{aligned}
$$


where

$$
\alpha(\mu)=\mu^{1 / 2}\left\{C_{4}(\mu)-\left(1+\pi^{2}\right) \mu\right\} .
$$

Since $C_{4}(\mu)=\left[2 \mu^{4} \pi^{2}\left(1+e^{-1 / \mu}\right)+\mu\left(1+\mu^{2} \pi^{2}\right)\right]\left(1+\mu^{2} \pi^{2}\right)^{-2}$, it is not difficult to show that for $\mu>0$, we have $\alpha(\mu)<0$.

Critical values for $U$ are obtained from the equation

$$
U\left[\alpha(\mu)\left\{1+\mu^{-5 / 2} a C_{2}(\mu) X U^{2}\right\}+g_{a} C_{1}(\mu) X U\right]=0,
$$

which gives

$$
\begin{aligned}
U^{(1)} & =0, \\
U^{(2,3)} & =\frac{\mu^{5 / 2}}{2 a \alpha(\mu) C_{2}(\mu)}\left[-g_{a} C_{1}(\mu) \pm \sqrt{\left\{g_{a} C_{1}(\mu)\right\}^{2}-\frac{4 a \alpha^{2}(\mu) C_{2}(\mu)}{\mu^{5 / 2} X(t)}}\right] .
\end{aligned}
$$

Since $\alpha(\mu)<0$ for $\mu>0$, the critical values $U^{(2,3)}$ are both positive provided

$$
\left\{g_{a} C_{1}(\mu)\right\}^{2}>\frac{4 a \alpha^{2}(\mu) C_{2}(\mu)}{\mu^{5 / 2} X(t)} .
$$

The integrals in (23) and (24) are evaluated to obtain

$$
\begin{aligned}
C_{1}(\mu) & =\int_{0}^{1} \varphi_{1}^{2} \tilde{\varphi}_{1}^{2} e^{-2 z / \mu} d z \\
& =\frac{\pi^{4} \mu^{7}\left(1-e^{-2 / \mu}\right)}{\left(1+\pi^{2} \mu^{2}\right)^{3}}+\frac{32 \pi^{4} \mu^{7}\left(1+e^{-1 / \mu}\right)}{\left(1+\pi^{2} \mu^{2}\right)^{3}\left(1+9 \pi^{2} \mu^{2}\right)}+\frac{\mu^{2}\left(\mu^{2} \pi^{2}+3\right)}{2\left(1+\pi^{2} \mu^{2}\right)^{2}}
\end{aligned}
$$

and

$$
\begin{aligned}
C_{2}(\mu) & =\int_{0}^{1} \varphi_{1}^{2} \bar{\varphi}_{1} \tilde{\varphi}_{1}^{2} e^{-2 z / \mu} d z \\
& =\frac{4 \sqrt{2} \pi^{3} \mu^{7}}{\left(1+\pi^{2} \mu^{2}\right)^{2}}\left\{\frac{\left(1-e^{-2 / \mu}\right)}{4\left(1+\pi^{2} \mu^{2}\right)}-\frac{4\left(1+e^{-2 / \mu}\right)}{\left(4+\pi^{2} \mu^{2}\right)\left(4+9 \pi^{2} \mu^{2}\right)}\right\} \\
& +\frac{32 \sqrt{2} \pi^{3} \mu^{7}\left(1+e^{-1 / \mu}\right)}{\left(1+\pi^{2} \mu^{2}\right)^{3}\left(1+9 \pi^{2} \mu^{2}\right)}+\frac{16 \sqrt{2} \pi^{3} \mu^{7}\left(1-e^{-1 / \mu}\right)}{\left(1+\pi^{2} \mu^{2}\right)^{2}\left(1+16 \pi^{2} \mu^{2}\right)}\left\{1-\frac{3}{1+4 \pi^{2} \mu^{2}}\right\} \\
& +\frac{4 \sqrt{2} \mu^{4}}{\pi\left(1+\pi^{2} \mu^{2}\right)^{2}}\left\{\frac{3}{8 \mu^{2}}+\frac{8}{15 \mu}+\frac{\pi^{2}}{8}\right\} .
\end{aligned}
$$

Since (27) implies that $X(t)$ is a strictly decreasing function of time, it is clear that if $X(t)$ is sufficiently small, $U^{(2,3)}$ become complex conjugates and (30) has only the real solution $U^{(1)}$. Writing

$$
\tilde{X}=\frac{4 a \alpha^{2}(\mu) C_{2}(\mu)}{\mu^{5 / 2}\left\{g_{a} C_{1}(\mu)\right\}^{2}}
$$


we see that if $X(0)<\tilde{X}, U(t)$ has only one critical value $U=0$. Thus, no matter how large $U(0)$ is, $U(t)$ decreases to zero as $t$ increases, indicating a diffusion type process.

For $X(0)>\tilde{X}$, let $U_{l}(t)$ and $U_{r}(t)$, denote $U^{(2,3)}(t)$, where $U_{l}(t)<U_{r}(t)$. Suppose we start with $U(0)$ such that $U_{l}(0)<U(0)<U_{r}(0)$. Then $\frac{d U}{d t}(0)>0$ and $U(t)$ increases toward $U_{r}(t)$. Meanwhile $X(t)$ is decreasing until at some $t$, say $t=t_{0}$, $X\left(t_{0}\right)=\tilde{X}$ and $U_{l}\left(t_{0}\right)$ and $U_{r}\left(t_{0}\right)$ coalesce. For $t>t_{0}, U^{(1)}$ is the only critical point and so $U(t)$ decreases to zero as $t$ increases. This proccess is typical of ignition, where the temperature of the medium increases to attain a maximum value and then decreases to zero due to the depletion of the medium.

Again, for $X(0)>\tilde{X}$, if $U(0)<U_{l}(0)$, then $U(t)$ is monotonically decreasing to zero. If $U(0)>U_{r}(0), U(t)$ decreases to $U_{r}(t)$ while $U_{r}(t)$ decreases and $U_{l}(t)$ increases. After some time $t$, say $t>t_{0}, U_{r}(t)$ and $U_{l}(t)$ become complex and $U(t)$ decreases to zero. In the first case, the initial temperature is too low to start an ignition. In the latter, the initial temperature is already high enough such that the burning of the medium does not have a boosting effect on the temperature. Both processes are of the diffusion type.

Taken together, the above results show that the value $U_{l}(0)$ serves as a critical initial condition below which ignition cannot occur. This notion of a critical initial condition for combustion problems has been investigated by Tam [5, 6], and Gray and Wake [3], among others.

\begin{tabular}{|cccc|}
\hline$\mu$ & $U_{l}(0)$ & \multicolumn{1}{c}{$U_{r}(0)$} & $\tilde{X}$ \\
\hline 0.061 & 6.015879 & 6.602364 & 8.983714 \\
0.065 & 4.785600 & 8.591181 & 8.274488 \\
0.100 & 3.217676 & 16.530145 & 4.911744 \\
0.150 & 2.832364 & 25.425909 & 3.247801 \\
0.200 & 2.808177 & 33.551855 & 2.566538 \\
0.250 & 2.916313 & 41.309390 & 2.218138 \\
0.300 & 3.093761 & 48.851314 & 2.017109 \\
0.400 & 3.567547 & 63.556698 & 1.812289 \\
\hline
\end{tabular}

TABLE 1. $U_{l}(0), U_{r}(0)$, and $\tilde{X}$ for different values of $\mu$.

Numerical results are obtained by taking $a=0.001, g_{a}=1.0, \lambda=1.0, \sigma_{a}=$ 0.001 , and $X(0)=20 \sqrt{2} / \pi$. Table 1 shows $U_{l}(0), U_{r}(0)$ and $\tilde{X}=4 a \alpha^{2}(\mu) C_{2}(\mu)$ $\mu^{-5 / 2}\left\{g_{a} C_{1}(\mu)\right\}^{-2}$ for different values of $\mu$.

For $\mu=0.1$, Figure 1 shows $U(t)$ for $U(0)=2.0,8.0$, and 20.0. The initial conditions are chosen to illustrate the three types of behaviour for $U(t)$. 


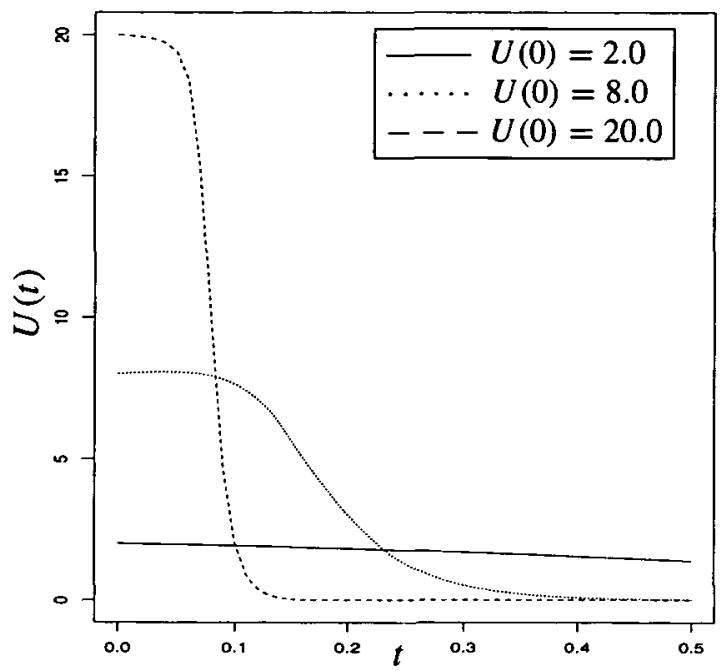

FIGURE 1. $U(t)$ as a function of $t$ for $U(0)=2.0,8.0$ and 20.0 with $\mu=0.1$.

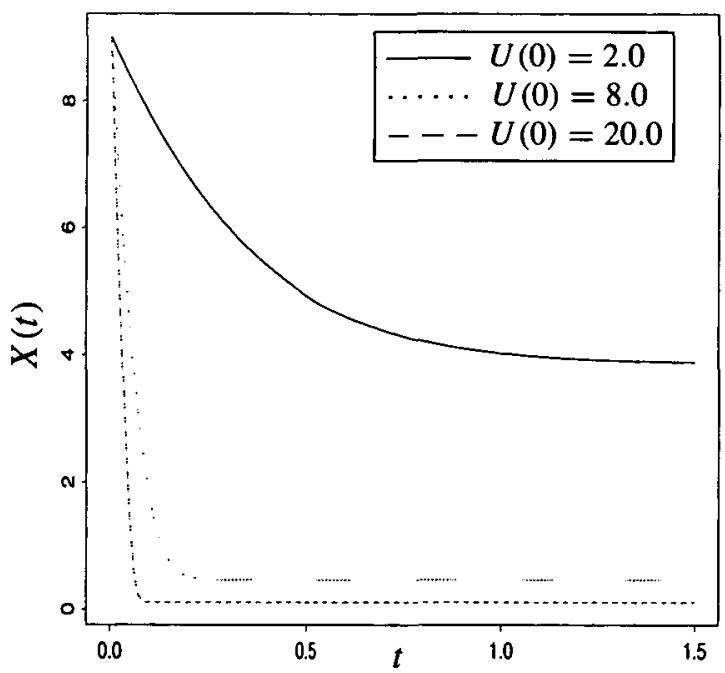

FIGURE 2. $X(t)$ as a function of $t$ for $U(0)=2.0,8.0$ and 20.0 with $\mu=0.1$.

In Figure 2, we present the graphs of $X(t)$ for those values of $U(0)=2.0,8.0$, and 20.0. It shows that a larger $U(0)$ gives rise to a smaller $\lim _{t \rightarrow \infty} X(t)$, suggesting more complete burning of the medium. 


\section{Numerical results for the truncated multi-mode system}

We consider the truncated multi-mode system

$$
\begin{aligned}
X_{n}^{\prime}= & -\mu^{-3 / 2} \lambda g_{a} \sum_{j=1}^{N} \sum_{k=1}^{N} \sum_{l=1}^{N} X_{j} U_{k} U_{l} \int_{0}^{1} \varphi_{j} \tilde{\varphi}_{k} \tilde{\varphi}_{l} \varphi_{n} e^{-2 z / \mu} d z \\
& -\mu^{-5 / 2} a \sum_{i=1}^{N} \sum_{j=1}^{N} \sum_{k=1}^{N} \sum_{l=1}^{N} X_{i}^{\prime} X_{j} U_{k} U_{l} \int_{0}^{1} \bar{\varphi}_{i} \varphi_{j} \tilde{\varphi}_{k} \tilde{\varphi}_{l} \varphi_{n} e^{-2 z / \mu} d z \\
\sigma_{a} U_{n}^{\prime} & +\sum_{i=1}^{N} \sum_{j=1}^{N} X_{i} U_{j}^{\prime} \int_{0}^{1} \varphi_{i} \varphi_{j} \varphi_{n} d z= \\
& -\gamma_{n}^{2} U_{n}+\mu^{-1} \sum_{i=1}^{N} U_{i} \int_{0}^{1} \tilde{\varphi}_{i} \varphi_{n} e^{-z / \mu} d z-U_{n}-\lambda^{-1} X_{n}^{\prime}
\end{aligned}
$$

where $N=3,5,9$, and 13 . Since computing time increases rapidly with increasing $N$, computation is carried out for $N=13$ only for a few values of the parameters. These results are then compared with the results for $N=1$.

In the following computations we have taken $a=0.001, g_{a}=1.0, \lambda=1.0$, and $\sigma_{a}=0.001$. The initial value is taken as $\chi(z, 0)=10$. By expanding $\chi$ in a Fourier sine series, is its $i^{\text {th }}$ coefficient $X_{i}(0)=20 \sqrt{2} /(i \pi)$. We shall use these values of $X_{i}(0)$ in the computation. We take first $u(z, 0)=U(0) \varphi_{1}(z)$.

Typical results are presented in Figures 3 and 4 . For $U(0)=2.0, u(z, t)$ is monotonically decreasing to zero as $t$ increases for $N=1,3,5$, and 9 . This phenomenon was predicted by the isolated fundamental-mode solution since $0.2<U_{l}(0)$. For $U(0)=8.0$, on the other hand, $u(z, t)$ increases to its maximum value and then decreases to zero as $t$ increases. This was again predicted by the isolated fundamentalmode solution since $U(0)=8.0$ is between $U_{l}(0)$ and $U_{r}(0)$. For $U(0)=4.0$ and $\mu=0.2$, Figure 5 gives $u(z, t)$ for $N=1,3,5$ and 9 . Since $U_{l}(0)<4.0<U_{r}(0)$, $u(z, t)$ increases toward its maximum and then decreases to zero, as predicted by the isolated fundamental-mode solution.

For $\mu=0.1$ and $U(0)=4.0$, we carry out a comparison of $\chi(1 / 2, t)$ for $N=$ 1, 3, 5, 9 and 13. The results are presented in Figure 6.

Some of the above computations are then repeated using the initial condition $u(z, 0)=8.0 \sin ^{2} 2 \pi z$, which is distinctly different from the first eigenfunction. Figure 7 shows that except in the time interval $0 \leq t \leq 0.15$, the first eigenmode again captures the essential behaviour of the solution as given by the nine-mode approximation. 

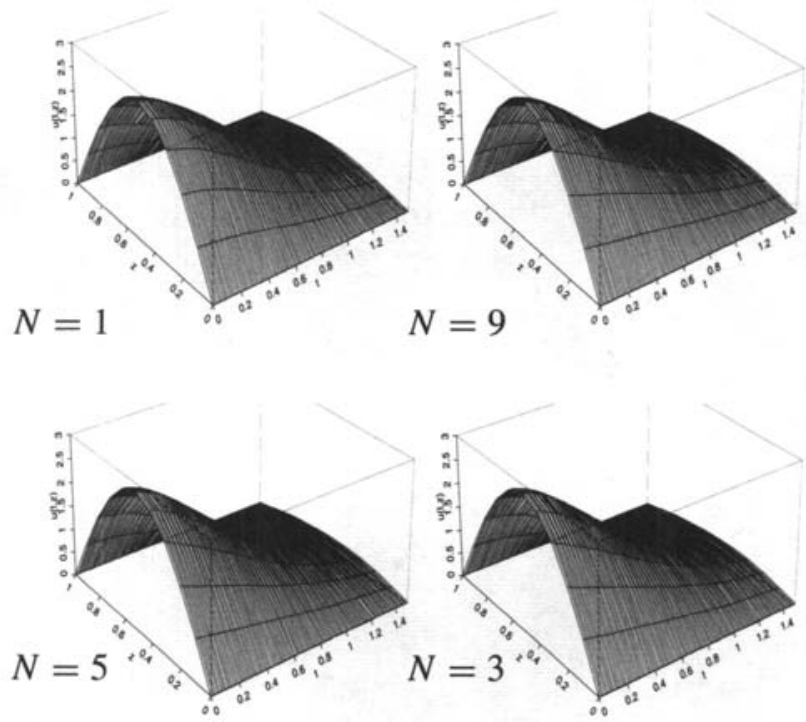

FIGURE 3. $u(z, t)$ with $\mu=0.1$ and $U(0)=2.0$ for $N=1,3,5$ and 9 .
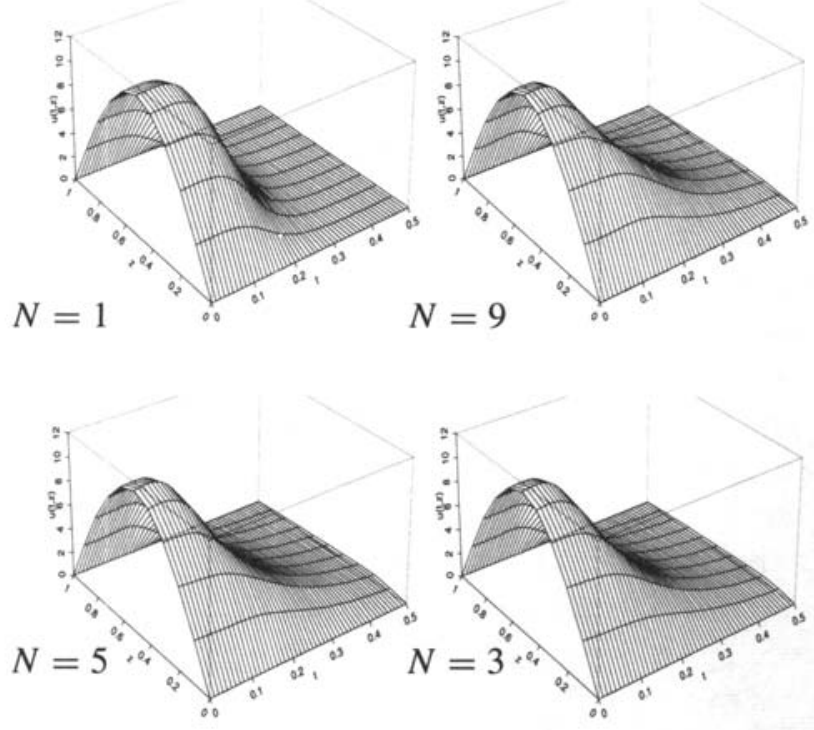

FIGURE 4. $u(z, t)$ with $\mu=0.1$ and $U(0)=8.0$ for $N=1,3,5$ and 9 . 

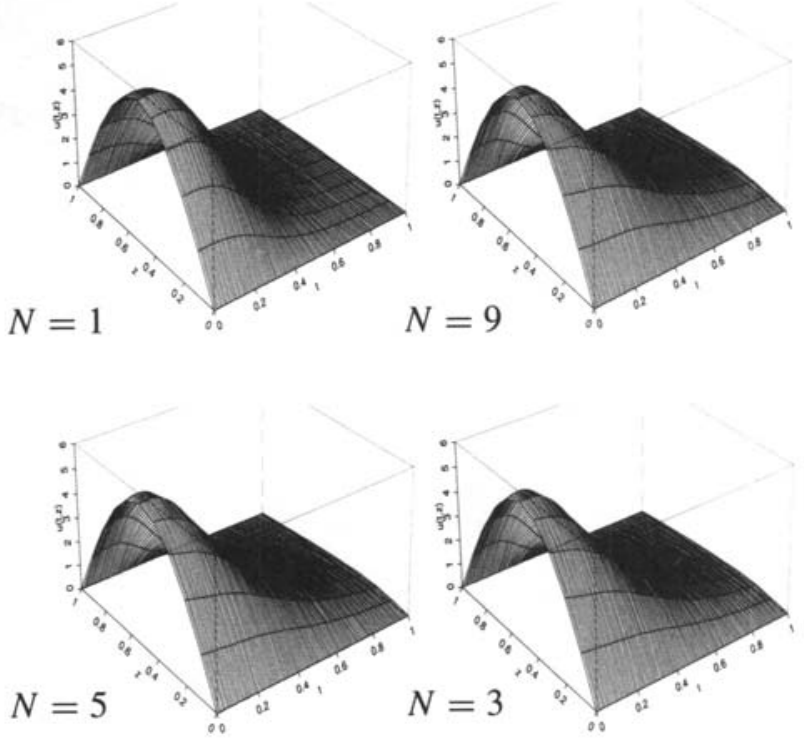

FIGURE 5. $u(z, t)$ with $\mu=0.2$ and $U(0)=4.0$ for $N=1,3,5$ and 9 .

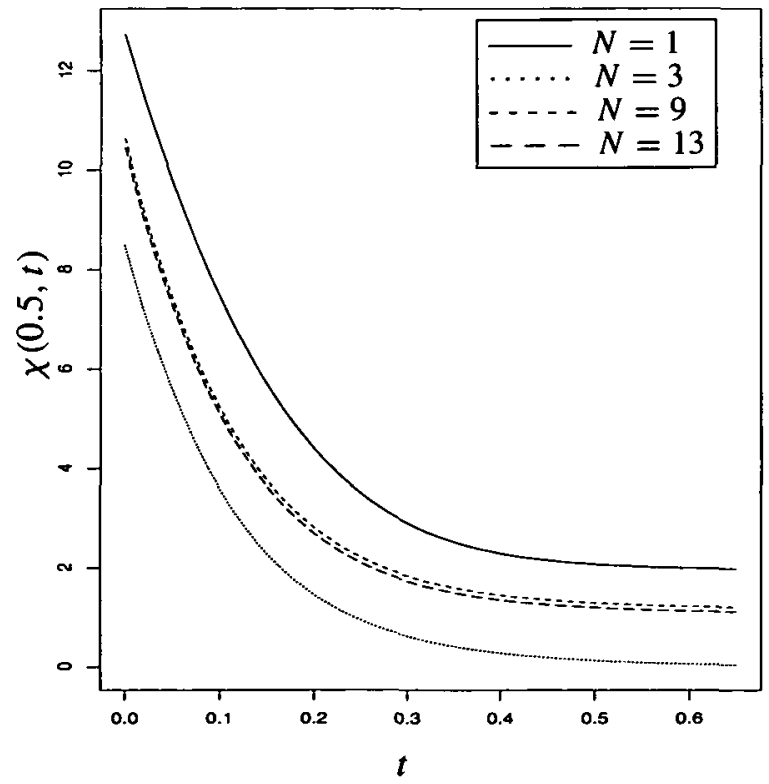

FIGURE 6. $\chi(0.5, t)$ with $U(0)=8.0$ and $\mu=0.1$ for $N=1,3,5$, and 9 . 

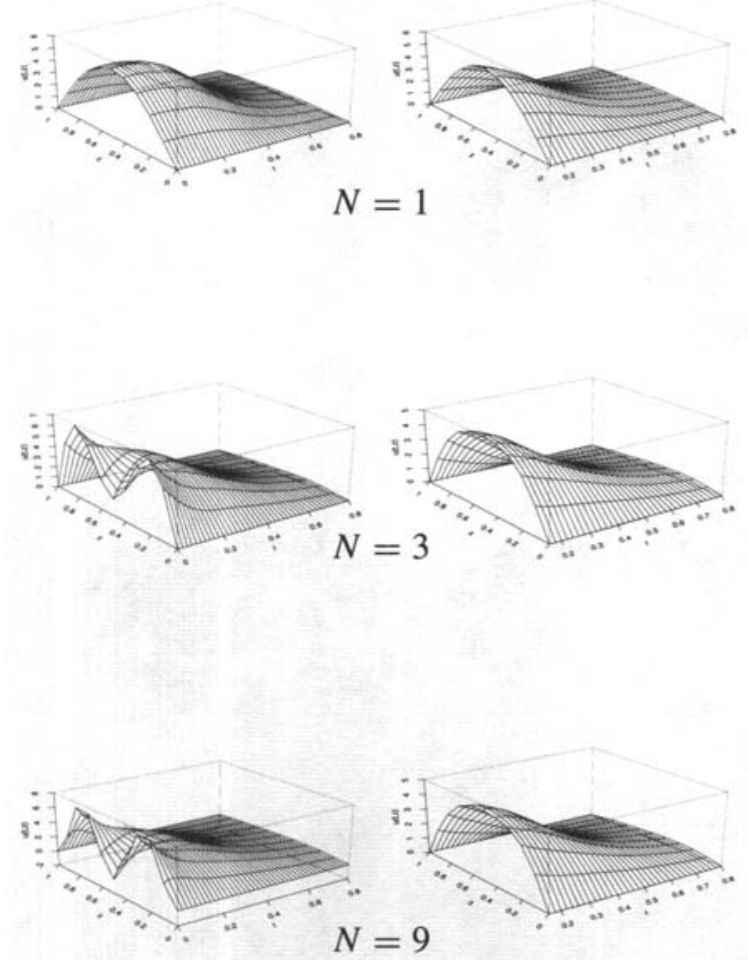

FIGURE 7. $u(z, t)$ with $\mu=0.2$ and $u(z, 0)=8.0 \sin ^{2} 2 \pi z$ for $N=1,3,5$, and 9 .

\section{Concluding remarks}

We have used eigenfunction expansions to construct the solution to the model problem of combustion of a porous slab. Computation using up to thirteen terms does confirm the conjecture that the salient features are captured by using the fundamental mode alone.

\section{Acknowledgement}

This research was partially supported by the Natural Science and Engineering Council of Canada. The second author (Andonowati) also acknowledges the financial support of the Indonesian Government. 


\section{References}

[1] D. S. Broomhead, R. Indik, A. C. Newell and D. A. Rand, "Local adaptive galerkin bases for large-dimensional dynamical systems", Nonlin. 4 (1991) 159-178.

[2] W. Echaus, Studies in Nonlinear Stability Theory (Springer, Berlin, 1965).

[3] B. F. Gray and G. C. Wake, "Critical initial conditions for thermal ignition", Math. and Comp. Modelling 18 (1993) 65-75.

[4] J. Norbury and A. M. Stuart, "A model for porous medium combustion", Quart. J. Mech. and Appl. Math. 42 (1989) 154-178.

[5] K. K. Tam, "Initial data and criticality for a problem in combustion theory", J. Math. Anal. Appl. 77 (1980) 626-634.

[6] K. K. Tam, "On the influence of the initial data in a combustion problem", J. Austral. Math. Soc. Ser. B 22 (1989) 193-209.

[7] K. K. Tam, "Porous medium combustion: ignition, temporal evolution, and extinction", J. Austral. Math. Soc. Ser. B 81 (1989) 249-263.

[8] R. Témam, Infinite Dimensional Systems in Mathematics and Physics, Volume 68 (Springer, Berlin, 1988). 